\title{
Reform der Rentenversicherung: Zuschussrente
}

\author{
Prof. Dr. Margarete Schuler-Harms \\ Vorsitzende der Kommission Recht der sozialen Sicherung, Fami- \\ lienlastenausgleich, Hamburg
}

Seit Herbst 2011 bemüht sich die Bundesregierung um eine Reform der Rentenversicherung. Ein Gesetzentwurf des Bundesministeriums für Arbeit und Soziales (BMAS) fasst verschiedene Änderungen im Rentenrecht und benachbarten Bereichen zusammen. Kern der Reform ist die sogenannte Zuschussrente. Das parlamentarische Verfahren läuft in der zweiten Jahreshälfte 2012 (im Juni 2012 war die Ressortabstimmung und die Prüfung der sich aus den Anhörungen von Verbänden, Ländern und Ressorts ergebenden Anregungen und Vorschlägen noch nicht abgeschlossen). Die Einführung der Zuschussrente soll ab 2013 und mit Übergangszeit erfolgen, während der erleichterte Zugangsbedingungen gelten. In Kürze lauten die vorgesehenen Regelungen ${ }^{1}$ :

- Wer 40 Versicherungsjahre sowie 30 Beitragsjahre aus Beschäftigung, Kindererziehung bis zum 10. Lebensjahr oder Pflege nachweist, erhält eine Zuschussrente. Die Zuschussrente ist der Betrag, um den die originäre Rente aufgestockt wird.

- Liegen diese Voraussetzungen vor, wird die Bewertung der Pflichtbeitragszeiten ab 1992 verdoppelt, jedoch bis auf maximal 1 Entgeltpunkt pro Jahr. Die Aufstockung ist zudem auf 31 Entgeltpunkte (West) bzw. 35 Entgeltpunkte (Ost) = aktuell jeweils etwa 850 Euro Bruttorente) begrenzt.

- Die Zuschussrente begünstigt nur Versicherte mit geringem Einkommen, indem zur Gewährleistung der Zielgenauigkeit eine Einkommensanrechnung erfolgt. Renten aus einer zusätzlichen Altersversorgung (betrieblich Altersvorsorge, Riester- und Rürup-Rente) stehen über die Zuschussrente hinaus voll zur Verfügung.

- Für Rentenansprüche, die ab 2019 entstehen, sind fünf Jahre an zusätzlicher Altersvorsorge erforderlich, um die Zuschussrente zu erhalten. Ab 2019 steigen die Anforderungen an den zeitlichen Umfang der zusätzlichen Altersvorsorge langsam und stetig von 5 auf 35 Jahre in 2049.

- Für Rentenansprüche, die ab 2023 entstehen, erhöht sich die Anzahl der erforderlichen Versicherungsjahre auf 45, in denen 35 Beitragsjahre aus Beschäftigung, Kindererziehung bis zum 10. Lebensjahr oder Pflege vorhanden sein müssen.

- Mit der Anknüpfung an die beitragsäquivalente Rente ist die Zuschussrente in größerem Umfang vorleistungsbezogen. Zu Beginn würden rund 52.000 Menschen von der Zuschussrente profitieren; 2030 könnten es etwa 1,4 Millionen sein.

I.

Am 16. April 2012 fand die Anhörung der Verbände zum Entwurf im BMAS statt. Der djb setzt sich schon seit vielen
Jahren für eine bessere Alterssicherung von Frauen und gegen die nachteiligen Folgen von Minijobs insbesondere für Frauen ein. Die djb-Kommission „Recht der sozialen Sicherung, Familienlastenausgleich “ - in der Anhörung vertreten durch die Vorsitzende Prof. Dr. Margarete Schuler-Harms, Hamburg und Prof. Dr. Astrid Wallrabenstein, Frankfurt - hat ausführlich schriftlich und mündlich zum Referentenentwurf eines Gesetzes zur Anerkennung der Lebensleistung in der Rentenversicherung (RV-Lebensleistungsanerkennungsgesetz) Stellung genommen. ${ }^{2}$

Fazit der im Folgenden referierten Stellungnahme ist: Die mit dem Gesetzentwurf zur Reform der Rentenversicherung verfolgten Ziele sind im Ganzen zu begrüßen. Dies gilt zunächst für die Verbesserungen bei der Bewertung der Zeiten für eine Erwerbsminderungsrente, die aus Sicht älterer Arbeitnehmerinnen und Arbeitnehmer einen engen Zusammenhang mit der Anhebung der Regelaltersgrenze bildet. Die Ungerechtigkeit der rentenrechtlichen Mechanik, nach der diese Rentenleistung gerade wegen der allmählichen Anhebung der Altersgrenze entwertet wurde, würde mit der vorliegenden Reform endlich behoben. Ebenso empfiehlt sich die in Aussicht genommene Flexibilisierung von Erwerbsarbeit und Alterssicherung in der Nähe der Regelaltersgrenze durch die bessere Möglichkeit einer Kombination von Erwerbseinkommen und Altersrente. Auch das Ziel, durch die Erweiterung der Möglichkeit zur Zahlung freiwilliger Beiträge die individuelle gesetzliche Rentenanwartschaft zu verbessern, ist begrüßenswert.

Die geplante Zuschussrente, die vor allem Frauen zugute kommen soll und zu der sich der djb deshalb ausführlicher äußert, verfolgt im Ganzen ebenfalls richtige Ziele (II.). In ihrer vorgesehenen Ausgestaltung und ohne weitere Maßnahmen, etwa ohne grundlegende Reformen im Bereich der geringfügigen Beschäftigung, dürfte die Zuschussrente diese Ziele allerdings nicht angemessen verwirklichen (III.).

Der djb empfiehlt deshalb:

1. auf die Einkommensanrechnung im Rentenfall (\$ 97a SGB VI-E) sowie

2. auf die Voraussetzung einer Gesamtversicherungszeit von 45 Jahren ( $\mathbb{S} 70 a$ Abs. 2 Nr. 1 SGB VI-E) bzw. 40 Jahren ( $(262$ a SGB VI-E) zu verzichten,

3. begleitend zur Einführung

a) die sozialversicherungsrechtliche Privilegierung geringfügiger Beschäftigung zu beseitigen

b) die Versicherungspflicht für selbständige Tätigkeit zu erweitern,

c) die dreijährige Erziehungszeit nach $\$ 56$ SGB VI auch auf vor 1992 geborene Kinder zu erstrecken sowie

d) die rentenrechtliche Absicherung der nicht erwerbsmäßigen Pflege zu verbessern und

\footnotetext{
1 Zitiert nach http://www.bmas.de/DE/Service/Presse/Pressemitteilungen/rentenreform-maerz-2012.html (Zugriff: 17.7.2012).

2 Sellungnahme vom... abrufbar, unter ....
} 
4. die Bindung an die Voraussetzung einer zusätzlichen Altersvorsorge ( $(70$ a Abs. 2 Nr. 3 SGB VI-E) zu überdenken. Im Einzelnen:

\section{Die Zielsetzungen der Zuschussrente}

\section{Minijob und Zuschussrente}

Der Ansatz des Gesetzentwurfs, die Frauen in der kontinuierlichen Ausübung einer sozialversicherungspflichtigen Erwerbstätigkeit zu stärken, ist uneingeschränkt zu begrüßen.

Vor allem zielt die Zuschussrente zu Recht auf eine Korrektur der rentenrechtlichen Folgen sog. „Minijobs“ und problematischer Effekte der Grundsicherung im Alter. Durch die in $\mathbb{S} \mathbb{S} 8,8$ a SGB IV geschaffene Möglichkeit werden in weit überdurchschnittlichem Maß Frauen dazu veranlasst, sozialversicherungsfreie Beschäftigung als Hauptberuf auszuüben. Dies ist keineswegs Zufall, sondern war vom Gesetzgeber von Anfang an so intendiert und ihm auch bei den Reformen von 2001, mit denen die Kombination von Entgelt- und zeitlicher Geringfügigkeit aufgehoben wurde, bewusst. Der Gesetzgeber sah und sieht ein arbeitsmarktpolitisches Bedürfnis für diese Beschäftigungsform als so gewichtig an, dass er es als Rechtfertigungsgrund dafür anerkennt, den Zugang zur Sozialversicherung in weitem Umfang zu verwehren. Da von dieser Möglichkeit insbesondere Frauen angesprochen seien, sei dies hinnehmbar, wenn und weil sie regelmäßig über ihre Ehepartner in die Sozialversicherung einbezogen (s. Familienversicherung in der GKV) und wirtschaftlich abgesichert (daher kein Bedarf an Arbeitslosenversicherung) seien. Die Nachteile in der gesetzlichen Rentenversicherung seien ebenfalls nicht so schwerwiegend. ${ }^{3}$ Die negativen Wirkungen, die mit der Ausweitung des Minijobs für die Sicherung des Lebensunterhalts im Alter einhergehen, versuchte der Gesetzgeber 2001 durch die Möglichkeit der freiwilligen Leistung von Rentenversicherungsbeiträgen (allein durch die Arbeitnehmerin) und für bestimmte Einkommenslagen durch die Einführung einer steuerfinanzierten Grundsicherung im Alter aufzufangen. Da die freiwillige Zahlung von Rentenbeiträgen nur geringe Rentenansprüche generiert ${ }^{4}$ und die Aussicht auf eine Grundrente der Bereitschaft zur Beitragszahlung im Niedriglohnsektor eher entgegenwirkt, ist es zu begrüßen, dass der vorgelegte Gesetzentwurf hier ansetzt.

Die Bestärkung der Frauen in der kontinuierlichen Ausübung einer Erwerbstätigkeit befördert auch zu Recht das Leitbild der erwerbstätigen Frau. ${ }^{5}$ Von einer zureichenden Sicherung im Alter können Frauen auch dann nicht länger ausgehen, wenn sie durch den Unterhalt des Ehe- oder Lebenspartners ausreichend gesichert scheinen: Im Falle der Arbeitslosigkeit bestehen im Rahmen des SGB II strenge Erwerbsobliegenheiten auch für den bislang nicht erwerbstätigen Partner. Auch bewirken die rechtlich wie kulturell erleichterte Möglichkeit der Scheidung und die (gesetzliche sowie richterrechtliche) Neugestaltung des Scheidungsunterhalts, dass auch verheiratete oder verpartnerte Frauen darauf eingestellt und in der Lage sein sollten, ggf. den eigenen Lebensunterhalt zu erwirtschaften. Der Gesetzgeber ist schon aufgrund der eigenen Entscheidungen gehalten, die bestehenden Anreize für ein wirtschaftliches riskantes Verhalten der Frauen nicht zu unterstützen, sondern vielmehr gegenläufige Anreize zu setzen.

Allerdings dürfte sich der Anreiz der Zuschussrente zu kontinuierlicher Erwerbstätigkeit und Altersvorsorge angesichts der starken Anreizwirkungen und negativen Effekte der geringfügigen Beschäftigung in Kombination insb. mit der beitragsfreien Familienkrankenversicherung als zu schwach erweisen. Der djb empfiehlt daher grundlegende Reformen im Bereich der geringfügigen Beschäftigung, die die Einführung der Zuschussrente flankieren. ${ }^{6}$

\section{Sonstige Zielsetzungen}

Die Zuschussrente knüpft an die Erwerbsbiografie an und ist damit „Rente“ im eigentlichen Sinn. Zugleich weist sie Aspekte des sozialen Ausgleichs insofern auf, als sie die aus diskontinuierlich verlaufenden Erwerbsbiografien im niedrigen bis mittleren Einkommensbereich resultierenden Defizite beim Alterseinkommen ausgleichend berücksichtigen möchte. Die Zuschussrente will dabei Wirkungen gerade bei Personen entfalten, welche mit Kindererziehung und Angehörigenpflege für das Rentensystem maßgebliche, bei der Bemessung des Alterseinkommens zu berücksichtigende Leistungen erbringen.

Problematisch ist unter diesem Aspekt die Einkommensabhängigkeit der Rentenleistung, die der djb schon deshalb nicht empfiehlt ${ }^{7}$ : Eine einkommensabhängige Leistung ist keine Anwartschaft im rentenrechtlichen Sinne, weil die Erfüllung der Anspruchsvoraussetzungen gerade noch nicht ausreichen soll, sondern die Leistung weiterhin bedingt bleibt. Die Einkommensanrechnung bei Hinterbliebenenrenten zeigt diesen Widerspruch: Hinterbliebenenrenten gelten - wie der Gesetzentwurf selbst betont - als fürsorgerisch motivierte Leistungen und gerade nicht als Honorierung eigener Lebensleistung, was ihre einkommensabhängige Ausgestaltung legitimiert. Wie dann aber eine einkommensabhängige Leistung als Honorierung eigener Lebensleistung verstanden werden kann, erschließt sich nicht.

Es ist daher zu erwarten, dass die Zuschussrente gerade nicht als Honorierung eigener Lebensleistung wahrgenommen wird, sondern als fürsorgerische Leistung innerhalb der Rentenversicherung, die eigentlich - wie die Grundsicherung im Alter - aus Steuermitteln zu finanzieren wäre. Die mit der Einkommensanrechnung geschaffene Unklarheit dürfte sowohl das „Image“ der Zuschussrente in der Bevölkerung prägen als auch ihre Anreizeffekte zunichtemachen (denn es ist nicht

3 Vgl. aktuell BSG, Urteil vom 15.7.2009, B 12 KR 14/o8 R, SGb 2010, 489 ff., mit Anm. Wallrabenstein mit unionsrechtlichen Bedenken.

4 Vgl. BMFSFJ, Neue Wege - gleiche Chancen. Gleichstellung von Frauen und Männern im Lebensverlauf, Erster Gleichstellungsbericht der Bundesregierung, vom 16.6.2011, BT-Drs. 17/6240, S. 210 und http://www.bmfsfj.de/BMFSFJ/Service/Publikationen/ publikationen,did=174358.html (Zugriff: 17.7.2011).

5 Zur Leitbildfrage und ihrer Bedeutung für die Gleichstellung von Frauen und Männern vgl. nur Gleichstellungsbericht 2011, S. 15.

6 S.u.III.1.

7 Zu weiteren Gründen vgl. unten 4- 
anzunehmen, dass eine junge Versicherte unter 30 Jahren für das Ziel, im Alter ihr Existenzminimum „+ 50 Euro“ von der Rentenversicherung anstatt vom Grundsicherungsträger zu erhalten, die Voraussetzungen erfüllen wird, für die die Zuschussrente Anreize setzen soll). Auch die spätere Einschätzung der Leistung durch die Rechtsprechung oder den ändernden bzw. korrigierenden Gesetzgeber ist angesichts dieser Ambivalenz der Leistung unsicher.

\section{Ausgestaltung der Zuschussrente}

An den Zielsetzungen gemessen zeigt die Ausgestaltung der Zuschussrente Licht und Schatten.

\section{Pflichtbeitragszeit von 35 bzw. 30 Jahren (\$70a Abs. 2 Nr. 2, § 262a SGB VI-E)}

Die langfristige Orientierung an einer Pflichtbeitragszeit von 35 Jahren erscheint wegen der Einrechnung von Beitrags- und Berücksichtigungszeiten für Kindererziehung und Zeiten der Angehörigenpflege grundsätzlich vertretbar. Frauenerwerbsbiografien weisen zwar zurzeit diese Pflichtversicherungszeiten noch nicht auf. Der Gesetzgeber darf jedoch durch entsprechende Anreize darauf hinwirken, dass solche Pflichtversicherungszeiten angestrebt werden.

Der djb empfiehlt, die Zuschussrente hierbei in ein Maßnahmensystem einzubinden, das insbesondere Frauen zu kontinuierlicher sozialversicherungspflichtiger Erwerbstätigkeit ermutigt, die mit dem Aufziehen von Kindern und der Pflege Angehöriger einhergehenden Bedarfe angemessen berücksichtigt und dadurch zum Bestand und zur Funktionsfähigkeit der Rentenversicherung beiträgt.

a) Weitere Maßnahmen für Kontinuität weiblicher Erwerbsbiografien erforderlich

Neben der Einführung der Zuschussrente empfiehlt sich die Prüfung und Durchführung weiterer Maßnahmen, mit denen die Kontinuität der weiblichen Erwerbsbiografie in Bezug auf die gesetzliche Rentenversicherung gefördert wird.

Es empfiehlt sich vor allem, die Einführung der Zuschussrente mit ernsthaften Bemühungen um die Beseitigung der sozialversicherungsrechtlichen Privilegierung geringfügiger Beschäftigung zu verbinden. Der Anreiz der Zuschussrente für kontinuierliche beitragspflichtige Erwerbstätigkeit würde, sofern es bei der bisherigen Ausgestaltung der geringfügigen Beschäftigung oder gar bei den bestehenden Reformplänen zu ihrer Ausweitung bliebe, im Lebensverlauf zu spät gesetzt. Auch die geplante Umkehrung des RegelAusnahme-Verhältnisses zugunsten der Rentenversicherungspflicht einer geringfügig Beschäftigten griffe noch zu kurz, denn sie würde den Arbeitnehmerinnen immer noch einen Optionenspielraum und damit die Möglichkeit belassen, den kurzfristigen finanziellen Einkommensvorteil der langfristigen und nach den geplanten Bezugsbedingungen keineswegs sicheren Zuschussrente im Alter vorzuziehen. Würde die privilegierte geringfügige Beschäftigung und damit dieser Spielraum auch noch ausgeweitet, dann wäre die Anreizwirkung der Zuschussrente durch einen solchen Beschäftigungsanreiz deutlich gemindert.

Die mit der Abschaffung der sozialversicherungsrechtlichen Privilegierung verbundene Förderung kleiner versicherungspflichtiger Teilerwerbstätigkeit wäre als kleineres Übel hinzunehmen, denn im Lebensverlauf hat sich vor allem der Übergang von der geringfügigen zur regulär sozialversicherungspflichtigen Beschäftigung für Frauen als hohe Hürde erwiesen. ${ }^{8}$

Schließlich ist auch die Einbeziehung selbständiger Tätigkeit in die Rentenversicherungspflicht jenseits der in $\mathbb{} 2$ Satz 1 SGB VI bereits erfassten Formen unter dem Aspekt der Pflichtversicherungszeit empfehlenswert.

Außerdem empfiehlt es sich, Zeiten der (einjährigen, versicherungspflichtigen) Arbeitslosigkeit als weitere Pflichtbeitragszeit zu berücksichtigen. Die Unterscheidung in „gute“, d.h. förderungswürdige, und „schlechte“ Pflichtbeitragszeiten ist verfassungsrechtlich fragwürdig.

b) Mehr Beitragszeiten für familiäre Sorgearbeit, insbesondere mehr Kindererziehungszeiten für vor 1992 geborene Kinder

Trotz Absenkung der erforderlichen Beitragszeit von 35 auf 30 Jahre wird diese Voraussetzung für rentennahe Jahrgänge besonders schwer zu erfüllen sein. Sie haben rollenbedingt weniger oft als die jüngeren Frauen einen Lebenslauf mit Versicherungszeiten aus Erwerbstätigkeit neben Kindererziehung und Angehörigenpflege aufzuweisen (wobei auch zu berücksichtigen ist, dass die Versicherungspflicht für selbständige Tätigkeit erst in den letzten Jahren ausgeweitet wurde) und erhalten für ihre zumeist vor 1992 geborenen Kinder jeweils nur ein Babyjahr angerechnet. Es empfiehlt sich deshalb, die Einführung einer Zuschussrente mit der Einführung der dreijährigen Kindererziehungszeit für Kinder, die vor 1992 geboren wurden, zu kombinieren. Ein Teil der Frauen wird dadurch gar keine Zuschussrente benötigen.

Auch die Berücksichtigung von Zeiten der Angehörigenpflege ist in den anstehenden Reformen der Pflegeversicherung auch unter dem Aspekt der Zuschussrente eingehender zu prüfen.

\section{Rentenrechtliche Zeiten von mindestens 45 Jahren ( $\$ 70 a$ Abs. 2 Nr. 1, § 262a SGB VI-E)}

Der djb empfiehlt weiter, auf das zusätzliche Erfordernis einer Gesamtbeitragszeit von 45 Jahren, für rentennahe Jahrgänge von 40 Jahren, zu verzichten. Durch dieses Erfordernis werden zu viele Frauen ausgeschlossen und der Zweck der Zuschussrente verfehlt. Eine rentenrechtliche Zeit von mindestens 45 Jahren ist für Frauen nur dann zu erreichen, wenn Zeiten wegen Kindererziehung und Angehörigenpflege mit Zeiten der Erwerbstätigkeit, der Arbeitslosigkeit und der freiwilligen Beitragszahlung glücklich kombiniert wurden und werden. Auch unterliegt diese Voraussetzung zu sehr den verfassungs-

8 Vgl. BMFSFJ, Erster Gleichstellungsbericht, BT-Drs. 17/6240, S. 130 ff., 155. 
rechtlich eröffneten Einflussmöglichkeiten des Gesetzgebers, der in der Vergangenheit z.B. erhebliche Einschnitte bei der Anrechnung von Ausbildungszeiten vorgenommen hat. Die Zuschussrente wird durch das Erfordernis der langen Versicherungszeit letztlich unkalkulierbar, mit negativen Folgen für ihre Funktion als Anreizinstrument.

\section{Mindestens 35 Jahre mit einer eigenständigen zusätzli- chen Altersvorsorge (\$ 70a Abs. $2 \mathrm{Nr} .3$ )}

Besonders dringlich erscheint dem djb der Verzicht auf das Erfordernis einer eigenständigen zusätzlichen Eigenvorsorge. Mit der Berücksichtigung von Eigenvorsorge als Anspruchsvoraussetzung der Zuschussrente wird für einen ganz bestimmten Personenkreis eine langjährige Selbstverpflichtung quasi-obligatorisch. Sie ist auch von Gewicht, da sie Sparleistungen von Menschen verlangt, die tendenziell keine oder wenig verfügbare Mittel hierfür haben. Eine solche Quasiverpflichtung bedarf zumindest sozialpolitisch - womöglich auch verfassungsrechtlich - der Rechtfertigung.

\section{a) Zuschussrentenniveau kein Anreiz für Eigenvorsorge}

Nach der Gesetzesbegründung soll mit dieser Regelung die Verbreitung von Eigenvorsorge für das Alter verbessert werden. Allerdings ist überaus zweifelhaft, dass sich mit dieser Regelung das genannte Ziel auch nur ansatzweise erreichen lässt. Die Annahme, dass Menschen mit Einkommen in der Nähe des Existenzminimums Eigenvorsorge betreiben und gewichtigen Verzicht leisten werden, um Jahrzehnte später die Aussicht auf Einkünfte in ungefähr der gleichen Höhe zu haben, ist sehr unrealistisch. Unterstellt man zudem rationales Verhalten, so wäre die konkrete Entscheidung, mit spätestens 30 Jahren einen sog. Riester-Vertrag mit dem Ziel abzuschließen, im Alter eine Zuschussrente zu erzielen, geradezu verfehlt. Denn hätten die betreffenden Frauen und Männer tatsächlich die Erwartung, während ihres gesamten zukünftigen Erwerbslebens so wenig zu verdienen, dass sie im Alter zuschussrentenberechtigt wären, dann wäre ihre gesamte Lebensplanung so prekär, dass sie langfristige vertragliche Zahlungsverpflichtungen gar nicht eingehen dürften. Umgekehrt ist die Bedienung eines Altersvorsorgevertrages in Zeiten nur geringen oder gar unzureichenden Einkommens nur dann rational, wenn man die Erwartung hat, später wieder so viel Einkommen zu haben, dass die Altersvorsorgebeträge nicht so gravierend ins Gewicht fallen. In diesem Fall ist man aber nicht Zielgruppe der Zuschussrente. Deshalb ist davon auszugehen, dass durch die Einführung der QuasiVerpflichtung zur Altersvorsorge in $\mathbb{5} 70$ a Abs. 2 Nr. 3 SGB VI-E die privatrechtliche Eigenvorsorge fürs Alter nicht, und zwar nicht einmal marginal, verbessert wird.

\section{b) Noch keine relevanten Alterseinkünfte}

Außerdem ist die Voraussetzung des $\mathbb{\$} 70$ a Abs. 2 Nr. 3 SGB VI-E so ausgestaltet, dass auch diejenigen, die sie erfüllen, also anreizkonform handeln, eine relevante Altersvorsorgeleistung nicht erwirtschaften. Pro Kalenderjahr muss nämlich nur ein Monat Eigenvorsorge für das Alter nachgewiesen werden, um die Anforderungen für die Zuschussrente zu erfüllen. Rational wäre daher eine Umsetzung dieses gesetzlichen Anreizes durch den Abschluss eines Riester-Vertrages, der nur einen Monat im Jahr bedient wird. Höhere Alterseinkünfte würden auf diese Weise nicht erreicht. Die gegenwärtige Konzeption der Zuschussrente kann das Grundproblem nicht lösen, dass für eine Verbesserung der Alterseinkünfte auf der Basis kapitalgedeckter Systeme einerseits die regelmäßige (!) Sparleistung über Jahrzehnte unverzichtbar ist, dass aber andererseits Menschen ohne ausreichendes Einkommen solche Sparleistungen gerade nicht erbringen können. Der Gesetzgeber kann zudem auch aus verfassungsrechtlichen Gründen eine Pflicht zum Sparen ohne Einkommen nicht errichten. ${ }^{9}$ Die Zuschussrente ist daher als Instrument zur Anregung von relevanter Eigenvorsorge völlig ungeeignet.

\section{c) Alleinerziehende}

Ergänzend ist zu bedenken, dass alleinerziehende Eltern es regelmäßig schwerer haben werden, Eigenvorsorge für das Alter zu betreiben, als Menschen, die zusammen mit einem Partner Kinder erziehen. Die Anforderungen müssten daher für Alleinerziehende wohl niedriger sein als für Verheiratete, für Menschen in Lebenspartnerschaft und solche in Lebensgemeinschaften. Dies würde aber verwaltungspraktische Probleme mit sich bringen, da dies beim Rentenzugang (eine frühere Kontenklärung findet nicht regelhaft statt) geprüft werden müsste.

\section{Anrechnung eigenen Einkommens im Alter (\$ $97 a$ SGB VI-E)}

Die Anrechnungsregelung, die sich schon für die Einordnung der Zuschussrente als Rentenleistung als problematisch erweist, bereitet auch in der Ausgestaltung unüberwindliche Schwierigkeiten.

\section{a) Nichtberücksichtigung von Einkommen aus nichteheli-} cher Lebensgemeinschaft (\$ 97a Abs. 1 SGB VI-E)

Die Beschränkung der Anrechnung auf eigenes Einkommen und auf Einkommen eines nicht getrennt lebenden Ehe- oder Lebenspartners ist im Hinblick auf den Schutz der Ehe verfassungsrechtlich bedenklich. Der Hinweis, bei der Ehe oder der eingetragenen Lebenspartnerschaft handele es sich um Einkommen aus einer Einstandsgemeinschaft, genügt zur Legitimation der Differenzierung nicht. Andererseits wäre eine volle Einkommensüberprüfung, wie sie bei der Bedarfsgemeinschaft nach $\int 9$ Abs. 2 SGB II erfolgt, für eine Rentenleistung, die als Ergebnis einer Lebensleistung konzipiert wird, ebenfalls nicht angemessen. Aus diesem Dilemma führt nur der Verzicht auf das Erfordernis der Einkommensanrechnung heraus.

\section{b) Privilegierung zusätzlicher Altersvorsorge} (\$ $97 a$ Abs. 2 S. 1 SGB VI-E)

Die Privilegierung von Einkommen aus zusätzlicher Altersvorsorge dient dem Ziel, in einem Rentenkorridor die Zuschuss-

9 BVerfGE 113, 1 - Kindererziehungszeiten in der Anwaltsversorgung. 
rente zu gewähren, in dem andernfalls gerade diese Eigenvorsorge zur Deckung des „zuschussrelevanten Existenzminimums" benötigt würde. Menschen, die die Voraussetzungen der Zuschussrente nicht erreichen und daher ggf. auf Grundsicherung im Alter angewiesen sind, müssen diese Einkünfte für die Sicherung ihres Existenzminimums verwenden. Motiv hierfür ist die Annahme, dass die Zielgruppe der Zuschussrente andernfalls gerade wegen der Zuschussrente den Anreiz zur Eigenvorsorge verliert. Allerdings besteht ein solcher Anreiz bisher nicht bzw. zu wenig - jedenfalls geht der Gesetzentwurf davon aus und erhebt u.a. eine 35-jährige Eigenvorsorge zur Zugangsvoraussetzung für die Zuschussrente. Also ist die Privilegierung dieser Einkünfte in $\mathbb{S} 97 \mathrm{a}$ Abs. 2 S. 1 Nr. 2 SGB VI-E letztlich eine Folgeregelung zu $\mathbb{S} 70$ a Abs. 2 Nr. 3 SGB VI-E.

Dies ist insgesamt kritikwürdig, weil Männer und Frauen zwar in gleichem Maße verpflichtet werden ( $\mathbb{S} 70 \mathrm{a}$ Abs. $2 \mathrm{Nr}$. 3 SGB VI-E), von der Privilegierung aber Männer weit mehr profitieren werden als Frauen. Die zweite und dritte Säule der Altersvorsorge wird deutlich mehr von Männern als von Frauen genutzt. Dies liegt insbesondere daran, dass betrieb- liche Altersvorsorge überwiegend in Branchen und Betrieben verbreitet ist, in denen überwiegend Männer beschäftigt sind. Zudem erfüllen Männer aufgrund ihres Erwerbsverhaltens (insb. weniger Teilzeit, weniger Erwerbsunterbrechungen, weniger Arbeitsplatzwechsel) eher die Voraussetzungen für betriebliche Altersvorsorge. Die strukturell höheren Einkommen von Männern ermöglichen zudem betriebliche und ebenso individuelle Altersvorsorge besser und in besserer Höhe. Im Ergebnis wird dies dazu führen, dass die Privilegierung dieser Einkommen im Rahmen der Zuschussrente vor allem Männern zugutekommen wird, während viele Frauen womöglich die Anforderungen des $\mathbb{5} 70$ a Abs. 2 Nr. 3 SGB VI-E zwar erfüllen, aber hieraus keine relevanten Alterseinkünfte erhalten werden. Die Zuschussrente wird also dazu führen, dass aus der Zielgruppe Frauen eher ausschließlich 850,- Euro und Männer eher 850,- Euro zuzüglich der Leistungen aus betrieblicher und individueller Altersvorsorge zur Verfügung haben werden. Es findet eine soziale Umverteilung in der gesetzlichen Rentenversicherung statt, für die typischerweise bei Männern höhere Einkommensgrenzen gelten als für Frauen.

\section{Aktionärinnen fordern Gleichberechtigung: Das Dogma von Qualifikation und Leistung - Einblicke in das djb-Projekt}

\section{Katrin Lange \\ Projektmitarbeiterin, Deutscher Juristinnenbund, Berlin}

Nach dem gelungenen Startschuss des Projekts „Aktionärinnen fordern Gleichberechtigung "1 am 19. März $2012^{2}$ besuchte der djb in diesem Jahr insgesamt 76 Hauptversammlungen börsennotierter Unternehmen und schloss mit dem Besuch der Hauptversammlung der Heidelberger Druckmaschinen AG am 26. Juli 2012 die bereits dritte Runde zur Erhebung frauenspezifischer Daten in der Privatwirtschaft erfolgreich ab. Insbesondere in der so genannten „heißen Phase“ im Mai fanden dabei mehrere Hauptversammlungen an einem Tag statt.

Konfrontiert wurden Aufsichtsräte und Vorstände in diesem Jahr unter anderem mit Fragen wie: „Welche Maßnahmen haben Sie ergriffen, damit künftig mehr Frauen für die Wahl zum Aufsichtsrat der Hauptversammlung vorgeschlagen werden können? Haben Sie sich konkrete Ziele gesetzt, auch im Sinne von einem Zeitrahmen, ob eine Frau in den nächsten fünf Jahren im Vorstand vertreten sein soll? Wie beabsichtigen Sie, die Lücke zwischen dem Anteil von Frauen an den Beschäftigten und dem Anteil von Frauen in Führungspositionen zu schließen? “. ${ }^{3}$

Die Reaktionen fielen dabei höchst unterschiedlich aus, selten jedoch sachlich: von Getuschel über Gelächter bis zu
Buh-Rufen, in den wenigsten Fällen blieb der Auftritt einer Teilnehmerin auf der Hauptversammlung unbemerkt. Doch nicht nur das Publikum scheint emotionalisiert beim Thema „Frauen in Führungspositionen“, auch der eine oder andere aus Aufsichtsrat und Vorstand ließ sich zu unsachlichen Antworten verleiten. Das Bedürfnis bei diesem Thema „etwas klarzustellen " ist enorm und kommt leider oft einer Art Zurechtweisung gleich. ${ }^{4}$

Doch auch die „sachlichen“ Antworten weisen auf ein grundlegendes Problem innerhalb der Debatte hin. Deutlich wird dies in dem oftmals vorgebrachten Argument, nicht Geschlecht, sondern Qualifikation und Leistung seien vorrangige Entscheidungsmerkmale für die Besetzung von Führungspositionen. In einer Gesellschaft, die auf dem Leistungsprinzip beruht, ist das nicht weiter verwunderlich. Allerdings werden dabei selten die Frauen und ihre Leistungen in den

\footnotetext{
1 Ausführliche Informationen zum Projekt auf der djb-Homepage: http://www.djb.de/Projekt_HV/.

2 An diesem Tag fand die Auftaktveranstaltung „Wie soll der Wandel gelingen?" statt. Der djb berichtete ausführlich in der Ausgabe 2/2012 der djbZ.

3 Fragenkatalog auf der djb-Homepage: http://www.djb.de/Projekt_ HV/HV2012-2013/Fragenkatalog_2012/.

4 Weitere Eindrücke der Teilnehmerinnen auf der djb-Homepage: http://www.djb.de/Projekt_HV/Presse/Stimmungsberichte/.
} 